\title{
Funciones de evaluación de calidad para un tránsito inteligente
}

\author{
Quality evaluation functions for smart transit
}

\author{
Laura Carballo, Andrea Villagra, Daniel Pandolfi \\ lcarballo@uaco.unpa.edu.ar,avillagra@uaco.unpa.edu.ar,dpandolfi@uaco.unpa.edu.ar \\ Laboratorio de Tecnologías Emergentes (LabTEm) \\ Instituto de Tecnología Aplicada (ITA) - Unidad Académica Caleta Olivia \\ Universidad Nacional de la Patagonia Austral
}

Recibido: 03/12/2020. Aceptado: 25/06/2021

\begin{abstract}
RESUMEN
Los problemas de tránsito aumentan constantemente y las ciudades del futuro solo pueden ser verdaderamente inteligentes si habilitan la Movilidad Inteligente (en inglés, Smart Mobility). Aplicaciones de movilidad inteligente como el control de semáforos, el estacionamiento inteligente y la gestión del tránsito, entre otras están comenzando a ser utilizadas en todo el mundo, trayendo beneficios a las ciudades, mejor calidad de vida, costos reducidos, uso de energía más eficiente y disminución de las emisiones vehiculares. Mejorar la movilidad representa un gran reto. Actualmente, una ciudad con tráfico congestionado y muchos embotellamientos conduce a más contaminación. Por lo tanto, con la gestión inteligente del tránsito, el consumo de combustible y las emisiones contaminantes pueden ser reducidas. A diferencia de técnicas tales como semáforos inteligentes que requieren nueva infraestructura, ubicación de sensores y modificaciones en obras civiles ya construidas, el uso de técnicas de inteligencia artificial en la optimización de los ciclos de semáforos se presenta como una herramienta viable, rápida, eficiente y de bajo costo. En este trabajo se proponen diferentes funciones de evaluación de calidad con un algoritmo genético celular (cGA) aplicadas a dos grandes escenarios cercanos a la realidad en áreas urbanas ubicadas en las ciudades de Málaga (España) y París (Francia), simuladas por el popular micro-simulador SUMO (Simulator Urban MObility). La comparación de las diferentes funciones revela la bondad de algunas funciones logrando a una reducción significativa en términos de las tasas de emisión y del consumo total de combustible, además de una reducción en los tiempos de variables de tránsito analizadas.
\end{abstract}

Palabras clave: Problema de planificación de luces de semafóricas; Algoritmo Genético Celular; Funciones de evaluación de calidad; Emisión de gases; Simulador SUMO.

\begin{abstract}
Traffic problems are constantly increasing and the cities of the future can only be truly smart if they enable Smart Mobility. Intelligent mobility applications such as traffic light control, intelligent parking, and traffic management, among others, are beginning to be used around the world, bringing benefits to cities, a better quality of life, reduced costs, more efficient energy use, and a decrease in vehicle emissions. Improving mobility represents a great challenge. Currently, a city with congested traffic and many traffic jams leads to more pollution. Therefore, with intelligent traffic management, fuel consumption, and polluting emissions can be reduced. Unlike techniques such as smart traffic lights that require new infrastructure, location of sensors, and modifications in civil works already built, the use of artificial
\end{abstract}


intelligence techniques in the optimization of traffic light cycles is presented as a viable, fast, efficient, and low cost. In this work, different quality evaluation functions are proposed with a cellular genetic algorithm (cGA) applied to two large scenarios close to reality in urban areas located in the cities of Malaga (Spain) and Paris (France), simulated by the popular SUMO micro-simulator (Simulator Urban MObility). The comparison of the different functions reveals the goodness of some functions, achieving a significant reduction in terms of emission rates and total fuel consumption, in addition to a reduction in the times of the analyzed transit variables.

Keywords: Traffic lights scheduling problem; Cellular Genetic Algorithm; Quality evaluation functions; Emission of gases; SUMO simulator.

\section{INTRODUCCIÓN}

En ciudades grandes son objetivos comunes controlar, disciplinar el tránsito y reducir accidentes en la ciudad, invirtiendo en sistemas de monitoreo y administración de tráfico. Los resultados que deben lograrse incluyen, por ejemplo, el uso de radares de velocidad, la programación adaptativa y en tiempo real de los semáforos, tomando en cuenta, entre otros factores, la concentración y el flujo de vehículos (dando prioridad a las ambulancias y los vehículos policiales y un corredor preferencial para colectivos), la concentración de peatones y la velocidad de los vehículos. Otra fuente de preocupación común es la oferta de sistemas de transporte público más eficiente, adecuado al desarrollo urbano y a la equidad social en relación con los desplazamientos. Muchas de las soluciones tienen el objetivo de preparar a la ciudad para la implementación, en el futuro, de un sistema multimodal de transporte, que incluya diferentes medios (bicicleta, subtes, franjas exclusivas para colectivos, vehículos livianos sobre rieles), y contribuya así a la reducción del consumo de combustibles, la emisión de gases y el tiempo de traslado, así como al mejoramiento de la calidad del aire.

Actualmente, una ciudad con tránsito congestionado y muchos embotellamientos conduce a más contaminación. Por lo tanto, con la gestión inteligente del tráfico se puede reducir el consumo de combustible y las emisiones contaminantes. A diferencia de técnicas tales como semáforos inteligentes que requieren nueva infraestructura, ubicación de sensores y modificaciones en obras civiles ya construidas, el uso de técnicas de inteligencia artificial en la optimización de los ciclos de semáforos se presenta como una herramienta viable, rápida, eficiente y de bajo costo (García-Nieto et al. 2014).

En este trabajo hemos tomado como inicio del análisis una función de evaluación de calidad (aptitud o fitness) utilizada en diferentes trabajos (García-Nieto et al. 2013, Villagra et. al, 2020) con algoritmos de inteligencia computacional, en particular utilizamos el cGA que ha tenido muy buenos resultados no solamente logrando una reducción en los tiempo de recorrido de los vehículos sino también logrando una reducción en los gases emitidos. Nuestro objetivo es analizar cómo afecta en los resultados obtenidos la modificación de la función de calidad, intentando obtener reducciones no solo en variables de tránsito sino también produciendo reducción en la emisión de gases y consumo de combustible.

El artículo está organizado de la siguiente manera: en la Sección 2 se describe el problema, en la Sección 3 se presenta una revisión de la literatura. En la Sección 4 se describen las funciones de evaluación propuestas. En la Sección 5 se especifican el algoritmo utilizado para resolver el problema, se explica cómo se representa el problema y la estrategia de solución. En la Sección 
6 se presenta el diseño de experimentos y en la Sección 7 se analizan los resultados. Finalmente, en la Sección 8 se describen las conclusiones y trabajos futuros.

\section{FORMULACION DEL PROBLEMA}

Una red de tránsito urbano está compuesta por intersecciones, luces de tráfico, rutas, direcciones y vehículos en movimiento sobre sus rutas. Las luces de tráfico están ubicadas en intersecciones y el control del flujo de los vehículos esta dado por sus programas de estados de color y tiempo de los ciclos (duración de las fases). Para todas las luces de tráfico ubicadas en una misma intersección la combinación de estados de color durante un ciclo es siempre válido y sigue las reglas de tráfico para intersecciones a fin de evitar colisiones.

Para describir matemáticamente el problema de estudio se emplea la representación utilizada por García-Nieto et al. (2013). Este problema se puede ver como un problema que tiene múltiples objetivos. Se pretende:

- maximizar el número de vehículos que llegan a su destino, $\mathrm{NV}_{D}$ o minimizar el número de vehículos que no llegan a destino, $\mathrm{NV}_{\mathrm{ND}}$, durante el tiempo de simulación $\mathrm{T}_{\mathrm{S}}$;

- minimizar el tiempo total de viaje de los vehículos, TTv (desde que arriba a la simulación hasta que llega a destino);

- minimizar el tiempo de paradas y de espera de todos los vehículos, TTEP

- maximizar el radio P de colores verde y rojo en cada estado de fase de todas las intersecciones, definida como:

$$
P=\sum_{i=0}^{i n t r} \sum_{j=0}^{f s} d_{i, j} \frac{v_{i, j}}{r_{i, j}}
$$

donde intr corresponde la cantidad de todas las intersecciones, $f s$ representa la cantidad de todas las fases, y $v_{i, j}, r_{i, j}$ representan el número de señales en color verde y color rojo, respectivamente, en la intersección $i$ y la fase $j$, con duración $d_{i, j}$.

Se combinan todos los objetivos en una única función objetivo representada de la siguiente forma:

$$
f_{o b j 1}=\frac{T T_{V}+T T_{E P}+N V_{N D} T_{S}}{N V_{D}^{2}+P}
$$

Las emisiones de los vehículos se ven influenciadas por la programación de los tiempos de los ciclos de las luces de tráfico (Kun y Lei, 2007).

Este problema de planificación de la duración de los ciclos semafóricos es complejo y presenta enormes espacios de búsqueda. Por ejemplo, una simple intersección con 8 fases de semáforo representa $55^{8}$ (más de $8,3 \times 10^{14}$ ) posibles soluciones. Además, se requiere del uso de simuladores para evaluar la calidad de las configuraciones de semáforos candidatas. Estos simuladores consumen mucho tiempo y es necesario contar con un algoritmo eficiente para la obtención de dichas soluciones. 


\section{REVISION DE LA LITERATURA}

Numerosos autores han considerado diversos aspectos de las intersecciones e intersecciones aisladas que forman parte de la coordinación de semáforos. Durante la última década, las técnicas de Inteligencia Computacional se han utilizado ampliamente para abordar este problema. Ceylan y Bell (2004) aplican un algoritmo genético (GA) para resolver el problema de asignación de tráfico y control de señal optimizando la sincronización de la señal. Sánchez et al. (2008) también aplicaron un GA, en una zona comercial de Santa Cruz (España), asumiendo que el tiempo de semáforo de cada intersección funciona independientemente de otras intersecciones. Otra investigación que utiliza un GA es el trabajo desarrollado por Dezani et al. (2012) que utiliza el algoritmo para resolver este problema aplicándolo a una red de tráfico urbano congestionado en un escenario en tiempo real con resultados prometedores. Pasando a otra metaheurística, Baskan y Haldenbilen (2008) y Baskan et al. (2011) implementan una variante de Optimización de Colonia de Hormigas (ACO) donde su enfoque se utiliza para el espacio de búsqueda de la solución potencial de un problema de optimización de tráfico de dos niveles. Jabbarpour et al. (2014) ofrecen una descripción general completa de los sistemas de control de congestión y enrutamiento de tráfico de vehículos basados en Colonia de Hormigas. En todos estos enfoques se optimizan los tiempos de viaje globales y el tiempo de espera de los vehículos en los semáforos, aunque ninguno de ellos tiene en cuenta las emisiones o el consumo de combustible.

Otra conocida metaheurística es la optimización de enjambres de partículas (PSO) que propone García-Nieto et al. (2012, 2013). Los principales objetivos de estos artículos fueron la maximización del número de vehículos que llegan a sus destinos, así como la minimización del tiempo total de viaje de los vehículos. Jun et al. (2012) proponen un método novedoso para el control de señales de tráfico utilizando el algoritmo PSO con Global Best Gradient Descent. Más recientemente, se han utilizado algoritmos PSO para detectar programas de ciclos de semáforos, con el objetivo de reducir el consumo de combustible y las emisiones vehiculares en áreas metropolitanas (Garcia-Nieto et al. 2014, Olivera et al. 2015). Souravlias et al. (2016) en un reciente artículo presentan un enfoque paralelo para el problema de la programación del semáforo utilizando Evolución Diferencial (DE), aplicado a escenarios de problemas cercanos a la realidad que consisten en dos grandes áreas urbanas ubicadas en las ciudades de Málaga (España) y París (Francia).

Se ha encontrado que los estudios de congestiones y contaminación que aplican enfoques multiobjetivos proporcionan resultados promisorios cuando se comparan con los algoritmos más avanzados (Zhouy Cai 2014, Péres et al. 2018, Nesmachnow et al. 2019).

El análisis de la literatura relacionada indica que las propuestas previas sobre la optimización del flujo y la contaminación de los semáforos utilizan una perspectiva uniobjetivo o multiobjetivo del problema, pero carecen de un análisis profundo de la función objetivo utilizada para lograr la solución al problema que está tratando de resolver. En este artículo proponemos nuevas funciones de evaluación de la calidad de las soluciones para determinar la que obtiene resultados precisos y produce la mayor reducción en la emisión de gases y consumo de combustible.

\section{FUNCIONES DE EVALUACION UTILIZADAS}

En esta sección, se describen las funciones de evaluación utilizadas para realizar este estudio. Se comienza con la función denominada $f_{o b j 2}($ Ecuación 3$)$. Esta función ha sido utilizada en 
Olivera et al. (2015), donde las variables principales de tráfico son las utilizadas en $f_{\text {objl }}$ (Ecuación 2) e incorpora información del consumo de combustible $(F C)$, y de gases emitidos como monóxido de carbono $(\mathrm{CO})$ y óxidos de nitrógeno $\left(N \mathrm{O}_{x}\right)$, como se muestra en la siguiente ecuación (Ecuación 3):

$$
f_{o b j 2}=\left(F C+C O+N O_{x}\right)+\left(w *\left(\frac{T T_{V}+T T_{E P}+N V_{N D} T_{S}}{N V_{D}^{2}+P}\right)\right)
$$

donde $w$ (con valor 0,5 ) se utiliza para mejorar el término ambiental del cálculo de toda la función de evaluación. El simulador utilizado (Simulator of urban mobility, SUMO, Krajzewicz, et al. 2006) es con el cual se obtiene la información de gases y combustible utilizado en la función.

Proponemos las siguientes versiones, que analizan cada una de las componentes que no son variables de tránsito de forma individual, dando lugar a las siguientes funciones de evaluación (Ecuaciones 4, 5 y 6):

$$
\begin{gathered}
f_{o b j 3}=C O \\
f_{o b j 4}=N O_{x} \\
f_{o b j 5}=F C
\end{gathered}
$$

Teniendo en cuenta que uno de los objetivos importantes es maximizar la cantidad de vehículos que llegan a su destino proponemos utilizar en las funciones anteriores esa variable y penalizar cuando no alcancen su destino. Las nuevas funciones de evaluación se presentan en las Ecuaciones 7,8 y 9.

$$
f_{\text {obj6 }}=C O+\left(N V_{N D} * k\right)
$$

donde $k$ se calcula como $\left.\left(\max (C O) / N V_{D}\right)^{*} 2\right)$.

$$
f_{o b j 7}=N O_{x}+\left(N V_{N D} * p\right)
$$

donde $p$ se calcula como $\left.\left(\max \left(N O_{x}\right) / N V_{D}\right)^{* 2}\right)$. 


$$
f_{o b j 8}=F C+\left(N V_{N D} * q\right)
$$

donde $q$ se calcula como $\left.\left(\max (F C) / N V_{D}\right) * 2\right)$.

En las siguientes tres Ecuaciones $(10,11$ y 12$)$ incorporamos más variables de tránsito a cada componente de gas emitido obteniendo las siguientes funciones de evaluación:

$$
\begin{gathered}
f_{o b j 9}=C O * f_{o b j 1} \\
f_{o b j 10}=N O_{x} * f_{o b j 1} \\
f_{o b j 11}=F C * f_{o b j 1}
\end{gathered}
$$

A continuación, añadimos otra componente de gas emitido generando las siguientes funciones de evaluación de las Ecuaciones 13 y 14:

$$
\begin{gathered}
f_{o b j 12}=\left(C O+N O_{x}\right) * f_{o b j 1} \\
f_{o b j 13}=(C O+F C) * f_{o b j 1}
\end{gathered}
$$

En la siguiente función de evaluación propuesta se pretende guiar la optimización en función del combustible utilizado dividido por el número de vehículos que llegan a destino (Ecuación $15)$ :

$$
f_{\text {obj14 }}=F C / N V_{D}
$$

Finalmente, se presenta una propuesta de normalización de la Ecuación 3 de la siguiente manera (Ecuación 16);

$$
f_{o b j 15}=\left(\frac{F C+C O+N O_{x}}{\max (F C)+\max (C O)+\max \left(N O_{x}\right)}\right)+\left(\frac{w * f_{o b j 1}}{N V * T_{S}}\right)
$$

donde $N V$ es el número de vehículos en la simulación y Ts es el tiempo de simulación.

Resumiendo, para este estudio se cuenta con 15 (quince) funciones de evaluación de calidad, la función original $\left(f_{o b j l}\right)$ que está formada únicamente por variables de tránsito, funciones que están formadas únicamente por variables de emisión de gases y combustible consumido $\left(f_{o b j 3}\right.$, 
$f_{o b j 4}$ y $\left.f_{o b j 5}\right)$ y el resto de las funciones que combinan variables de tránsito, gases y combustible $\left(f_{o b j}, f_{o b j 6}, f_{o b j}, f_{o b j 8}, f_{o b j}, f_{o b j 10}, f_{o b j 11}, f_{o b j 12}, f_{o b j 13}, f_{o b j 14}\right.$ y $\left.f_{o b j 15}\right)$.

\section{ALGORITMO, REPRESENTACION Y ESTRATEGIA DE SOLUCION}

En esta sección se presenta el algoritmo utilizado en este trabajo (cGA) para realizar el análisis de las diferentes funciones propuestas, la representación y estrategia de solución.

\subsection{Descripción de cGA}

El cGA es una clase particular de Algoritmo Evolutivo (EA) y una subclase de los Algoritmos Genéticos con una población estructurada espacialmente, es decir, los individuos de la población pueden aparearse con sus vecinos (Dorronsoro y Alba, 2008). Estos pequeños vecindarios solapados ayudan a explorar el espacio de búsqueda debido a una difusión lenta de las soluciones permitiendo la exploración y una explotación que se lleva a cabo en cada vecindario a través de los operadores genéticos. En un cGA la población está usualmente estructurada en una rejilla toroidal de 2 dimensiones. Como familia de un GA, el cGA codifica las variables de decisión de un problema de búsqueda en cadenas de variables de longitud finita de algún alfabeto de cierta cardinalidad. Las cadenas son soluciones candidatas y se llaman cromosomas. De la misma forma que en un GA a cada una de las variables que forman el cromosoma se las denomina gen y alelo a los distintos valores que pueden tomar los genes. Codificado el problema que resolver a través de uno o varios cromosomas (también llamados individuos) y teniendo definida la función de aptitud, se evolucionan las soluciones al problema, es decir la población de soluciones teniendo en cuenta los siguientes pasos: Inicialización, evaluación, selección, recombinación, mutación y reemplazo.

\subsection{Representación y estrategia de solución}

Una posible solución al problema se representa por medio de un vector de enteros positivos, cada número entero representa la duración en segundos para cada fase de cada intersección dentro del ciclo semafórico. En este contexto del problema a resolver, el principal objetivo consiste en encontrar programas de ciclos optimizados para todos los semáforos situados en una determinada área urbana. Se define un ciclo como el periodo de tiempo en el que un conjunto de semáforos (en un cruce) permanecen con sus estados de luces/colores fijos. Al mismo tiempo, estos programas deben también coordinarse con semáforos en intersecciones adyacentes, mejorando así el flujo de vehículos que circulan.

En cuanto a la representación como se dijo anteriormente y siguiendo la especificación de SUMO para la programación de ciclos, se codifican las posibles soluciones como vectores de enteros. De esta forma, cada elemento del vector (variable) representa una duración de fase de los semáforos implicados en una determinada intersección

La estrategia de optimización se muestra en la Figura 1. Cuando el cGA genera una nueva solución, esta es inmediatamente utilizada para actualizar el programa de ciclos, entonces se ejecuta SUMO, para simular el escenario de una instancia con calles, direcciones, obstáculos, luces de tráfico, vehículos, rutas, etc. Con el nuevo estado del programa de ciclos. Luego de la simulación, SUMO devuelve la información global necesaria para calcular la calidad de la solución (fitness). Además, devuelve información de los siguientes gases $\mathrm{CO}_{2}$ (dióxido de 
carbono), $\mathrm{CO}$ (monóxido de carbono), $\mathrm{NO}_{\mathrm{x}}$ (óxidos de nitrógeno), $\mathrm{PM}_{\mathrm{x}}$ (materia particulada) y $\mathrm{HC}$ (hidrocarburos).

Dentro de los gases generados en la combustión, no todos son nocivos para salud. En particular, los gases emitidos por un motor de combustión interna de gasolina son, principalmente, de dos tipos: inofensivos y contaminantes. Los primeros están formados, fundamentalmente, por Nitrógeno, Oxígeno, Dióxido de Carbono, vapor de agua e Hidrógeno. Los segundos o contaminantes están formados, fundamentalmente, por el Monóxido de Carbono, Hidrocarburos, Oxidos de Nitrógeno y materia Particulada.

$\mathrm{El} \mathrm{CO}_{2}$ se produce cuando se queman combustibles que contienen carbono (por ejemplo, gasolina, combustible diesel). El carbono se combina durante esta operación con el oxígeno aspirado. $\mathrm{El} \mathrm{CO}_{2}$, a pesar de ser un gas no tóxico, reduce el estrato de la atmósfera de la Tierra que generalmente sirve como protección contra la penetración de los rayos UV (la tierra se calienta), produce alteraciones de clima (efecto "invernadero").

El CO se produce debido a la combustión incompleta de carbono que contiene y combustibles altamente tóxicos. Es un gas incoloro, inodoro, explosivo y altamente tóxico. Bloquea el transporte de oxígeno por parte de los glóbulos rojos. Es mortal, incluso en una baja concentración en el aire que respiramos. En una concentración normal en el aire ambiental se oxida al corto tiempo, formando dióxido de carbono $\mathrm{CO}_{2}$.

Los $\mathrm{NO}_{\mathrm{x}}$ son combinaciones de nitrógeno $\mathrm{N}_{2}$ y oxígeno $\mathrm{O}_{2}$ (por ejemplo, $\mathrm{NO}, \mathrm{NO}_{2}, \mathrm{~N}_{2} \mathrm{O}$ ). Los óxidos de nitrógeno se producen al existir una alta presión, alta temperatura y exceso de oxígeno durante la combustión en el motor. El monóxido de nitrógeno (NO), es un gas incoloro, inodoro e insípido. Al combinarse con el oxígeno del aire, es transformado en dióxido de nitrógeno $\left(\mathrm{NO}_{2}\right)$, de color pardo rojizo y de olor muy penetrante, provoca una fuerte irritación de los órganos respiratorios. Las medidas destinadas a reducir el consumo de combustible suelen conducir lamentablemente a un ascenso de las concentraciones de óxidos nítricos en los gases de escape, porque una combustión más eficaz produce temperaturas más altas. Estas altas temperaturas generan a su vez una mayor emisión de óxidos nítricos.

Los $\mathrm{HC}$ son restos no quemados del combustible, que surgen en los gases de escape después de una combustión incompleta. La mala combustión puede ser debido a la falta de oxígeno durante la combustión (mezcla rica) o también por una baja velocidad de inflamación (mezcla pobre), por lo que es conveniente ajustar la riqueza de la mezcla. Los hidrocarburos $\mathrm{HC}$ se manifiestan en diferentes combinaciones y actúan de diverso modo en el organismo. Algunos de ellos irritan los órganos sensoriales, mientras que otros son cancerígenos.

$\mathrm{PM}_{\mathrm{x}}$ es generada en su mayor parte por los motores diesel, se presentan en forma de hollín o cenizas. Los efectos que ejercen sobre el organismo humano todavía no están aclarados por completo. 


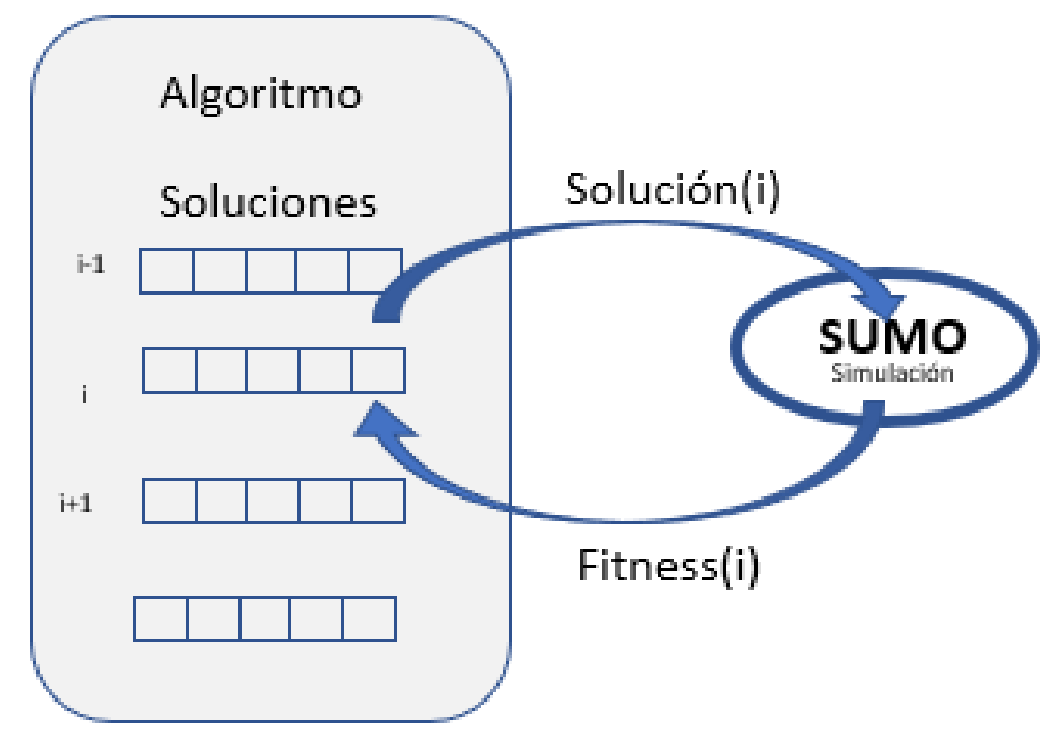

Figura 1: Estrategia de optimización. El algoritmo invoca a SUMO para evaluar cada solución

En esta sección se presentan características de las instancias utilizadas, la parametrización de cGA y el diseño de los experimentos.

Para analizar las diferentes funciones de evaluación se utilizaron dos instancias de problemas ubicadas en dos grandes áreas metropolitanas (Málaga y Paris). Las instancias consideradas fueron creadas a partir de la extracción de información de mapas digitales reales. La instancia de Málaga cuenta con 190 luces de tráfico y la instancia de parís con 378 luces de tráfico.

En cuanto a cGA la parametrización la siguiente: (a) el tamaño de la población es de 100 soluciones (individuos); (b) para la recombinación, uno de los padres es el individuo actual considerado en el bucle de cGA, mientras que el otro es obtenido utilizando la Selección de torneos en su vecindario; (c) el método de recombinación es DPX1 (crossover de dos puntos), es cual produce un solo individuo (que contiene la porción más grande del mejor padre), con una aplicación de probabilidad establecida en 1.0; (d) la mutación utilizada es entera (intercambio de un bit) con una probabilidad de establecida en 1 / L donde $\mathrm{L}=$ longitud de la solución. Finalmente, (e) el criterio de reemplazo es "Si no es peor" con un reemplazo síncrono. El número máximo de evaluaciones fue establecido en 30.000. Los valores utilizados se han establecido en base a experimentaciones realizadas y de trabajos anteriores donde se han utilizado los algoritmos (Villagra et al., 2020).

Respecto al procedimiento de simulación, cada vehículo inicia su propio viaje desde un punto de partida hasta un destino con una velocidad máxima de $50 \mathrm{~km} / \mathrm{h}$. Este límite de velocidad es típico en áreas urbanas. El tiempo de simulación se fijó en 2.200 segundos para la instancia Málaga y 3.400 segundos para la instancia de París, ya que es de mayor tamaño. La simulación se realizó ejecutando el Simulador de tráfico SUMO versión 0.31.0 para Linux.

Todos los algoritmos se implementaron en Java y se ejecutan en el clúster que consta de 16 nodos (64 núcleos) equipados con una CPU IntelCore2 Quad (Q9400) @ 2.66 GHz y 4 GB de RAM, 14 nodos (28 núcleos) equipados con Intel Pentium D a 2,8 GHz y 1 GB de RAM, un nodo (ocho núcleos) equipado con dos Intel Xeon (E5405) a $2.00 \mathrm{GHz}$ y 8 GB de RAM, y un nodo (ocho núcleos) equipado con un Intel Core i7 (920) a $2.67 \mathrm{GHz}$ y 4 GB de RAM. El 
software responsable de la gestión de estos recursos heterogéneos es HTCondor Versión 7.8.4 (Thain et al., 2005). Se realizaron 30 ejecuciones independientes para cada función de evaluación con cada una de las instancias. En este trabajo, los mejores valores están marcados en negrita. En todos los experimentos se han analizado las condiciones que deben cumplirse para utilizar pruebas paramétricas y pruebas no paramétricas para el análisis estadístico con un error de probabilidad de $p=0,05$ utilizando RStudio Versión 1.1.463. Las diferencias estadísticamente significativas entre los algoritmos/funciones se muestran con el símbolo $(\bullet)$, mientras que la no significación se muestra con (-). Además, se utiliza el símbolo $(\rightarrow)$ para nombrar entre quienes existe diferencia estadísticamente significativa.

Para realizar el estudio de las diferentes funciones el experimento realizado está compuesto por cuatro etapas. Teniendo en cuenta que para realizar los análisis de variables de tránsito y emisiones de gases y combustible consumido obtenido por cada una de las funciones es necesario que todos los vehículos utilizados en la simulación alcancen su destino, la primera etapa consiste en seleccionar todas las funciones que cumplan con esta condición. Como segunda etapa se comparan las funciones seleccionadas en la primera etapa y se analizan los resultados obtenidos en cuanto a variables de tránsito. Como tercera etapa se comparan las funciones seleccionadas en la primera etapa atendiendo a los resultados obtenidos en cuanto a variables de gases y combustible. Finalmente, la cuarta etapa consiste en analizar los resultados obtenidos por las funciones que obtienen los mejores resultados en la segunda y tercera etapa.

\section{ANALISIS DE RESULTADOS}

En esta sección se presentan y analizan los resultados obtenidos por los estudios realizados en cada una de las etapas definidas en el experimento.

En la Tabla 1 se muestran los resultados obtenidos en media por cada función en cuanto a las variables de tránsito, número de vehículos que llegan a destino $\left(\mathrm{NV}_{\mathrm{D}}\right)$ y número de vehículos que no llegan a destino ( $\mathrm{NV}_{\mathrm{ND}}$ ), para las instancias de Málaga y de Paris. La primera columna corresponde a cada función de evaluación y las cuatro siguientes columnas corresponden a algunas componentes utilizadas en algunas funciones de evaluación y descriptas en las Sección 2. Podemos observar en color rojo las funciones donde no se llegó a que la totalidad de vehículos alcancen su destino $\left(f_{o b j 2,} f_{o b j 3,}, f_{o b j 4,} f_{o b j 5,} f_{o b j 14}\right.$ y $\left.f_{o b j 15}\right)$ tanto para la instancia de Málaga y de Paris. Las funciones $\boldsymbol{f}_{\boldsymbol{o b j}} \mathrm{y}$ y $\boldsymbol{f}_{\boldsymbol{o b j}}$ únicamente para la instancia de Málaga hay vehículos que no llegan a destino. Las funciones para las cuales todos los vehículos han llegado a su destino para ambas instancias (Málaga y Paris) son siete de las quince funciones y están resaltadas en

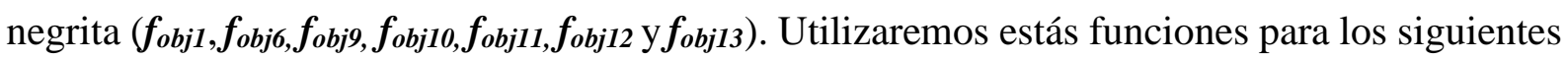
análisis (segunda etapa). 
Tabla 1: Mediana de resultados obtenidos por las funciones propuestas para la instancia de Málaga y de Paris

\begin{tabular}{|c|c|c|c|c|}
\hline \multirow[b]{2}{*}{ Función } & \multicolumn{2}{|c|}{ Málaga } & \multicolumn{2}{|c|}{ Paris } \\
\hline & $\mathbf{N V}_{\mathbf{D}}$ & $\mathbf{N V}_{\text {ND }}$ & $\mathbf{N V _ { D }}$ & $\mathbf{N V}_{\text {ND }}$ \\
\hline$f_{o b j 1}$ & 1200 & 0 & 1200 & 0 \\
\hline$f_{o b j 2}$ & 405 & 795 & 625 & 575 \\
\hline$f_{o b j 3}$ & 431 & 769 & 607 & 593 \\
\hline$f_{o b j 4}$ & 431 & 769 & 585 & 615 \\
\hline$f_{o b j 5}$ & 374 & 826 & 529 & 672 \\
\hline$f_{\text {obj6 }}$ & 1200 & 0 & 1200 & 0 \\
\hline$f_{o b j}$ & 1199 & 1 & 1200 & 0 \\
\hline$f_{o b j 8}$ & 1199 & 1 & 1200 & 0 \\
\hline$f_{\text {obj } 9}$ & 1200 & 0 & 1200 & 0 \\
\hline$f_{o b j 10}$ & 1200 & 0 & 1200 & 0 \\
\hline$f_{\text {obj } 11}$ & 1200 & 0 & 1200 & 0 \\
\hline$f_{o b j 12}$ & 1200 & 0 & 1200 & 0 \\
\hline$f_{\text {obj13 }}$ & 1200 & 0 & 1200 & 0 \\
\hline$f_{o b j 14}$ & 922 & 278 & 922 & 278 \\
\hline$f_{o b j 15}$ & 424 & 777 & 424 & 777 \\
\hline
\end{tabular}

En la Tabla 2 se muestran las medianas de los resultados obtenidos para variables de tránsito (tiempo total de viaje de los vehículos, TTV y tiempo de paradas y de espera de todos los vehículos, $\left.\mathrm{TT}_{\mathrm{EP}}\right)$ con las funciones seleccionas $\left(\boldsymbol{f}_{\boldsymbol{o b j}}, \boldsymbol{f}_{\boldsymbol{o b j}}, \boldsymbol{f}_{\boldsymbol{o b j}}, \boldsymbol{f}_{\boldsymbol{o b j 1 0}}, \boldsymbol{f}_{\boldsymbol{o b j} \mathbf{1 1}}, \boldsymbol{f}_{\boldsymbol{o b j} 12}\right.$ y $\left.\boldsymbol{f}_{\boldsymbol{o b j 1 3}}\right)$ para las instancias de Málaga y de Paris. La función $f_{\text {objl }}$ es la que obtiene los menores valores para ambas variables. Realizando las pruebas estadísticas correspondientes (Shapiro para determinar normalidad, Levene para determinar homocedasticidad y Kruskall-Wallis (K-W) o ANOVA, según corresponda, para determinar diferencias estadísticamente significativas entre los resultados obtenidos por las funciones) los resultados muestran que existen diferencias estadísticamente significativas entre los resultados de las funciones para las variables analizadas $\left(\mathrm{TT}_{\mathrm{v}}\right.$ y $\mathrm{TT}_{\mathrm{EP}}$ ), en ambas instancias. La fila denominada Holm presenta los resultados de aplicar el test post-hoc de Holm a los resultados de las funciones. Encontramos que existen diferencias estadísticamente significativas únicamente entre $\boldsymbol{f}_{\boldsymbol{o b j} \mathbf{l}}$ y $\boldsymbol{f}_{\boldsymbol{o b j} \boldsymbol{b}}$, con la instancia de Málaga y para la instancia de Paris las diferencias estadísticamente significativas están presentes entre $\boldsymbol{f}_{\boldsymbol{o b j} \mathbf{1}}$ con respecto a $\boldsymbol{f}_{\boldsymbol{o b j} \boldsymbol{6}} \mathrm{y}$ con respecto a $\boldsymbol{f}_{\boldsymbol{o b j 1 0}}$. Esto se ve reflejado en la donde podemos observar en las gráficas (a) y (b) el boxplot correspondiente a los resultados obtenidos por las funciones para la variable $\mathrm{TT}_{\mathrm{v}}$ y la clara diferencia entre las medianas de las funciones $\boldsymbol{f}_{\boldsymbol{o b j} \mathbf{1}}$ y $\boldsymbol{f}_{\boldsymbol{o b j} \boldsymbol{6}}$ además de las distribuciones de los resultados alrededor de su mediana.

Para la variable tiempo total de espera $\left(\mathrm{TT}_{\mathrm{EP}}\right)$ los resultados obtenidos por la función $\boldsymbol{f}_{\boldsymbol{o b j}}$ presenta diferencias estadísticamente significativas con respeto a cuatro funciones en cuanto $\left(f_{o b j 6}, f_{o b j 10}, f_{o b j 11}\right.$ y $\left.f_{o b j 13}\right)$ con la instancia de Málaga. Mientras que para la instancia de Paris la función $\boldsymbol{f}_{\boldsymbol{o b j} \mathbf{1}}$ tiene diferencias estadísticamente significativas solamente con dos funciones $\left(\boldsymbol{f}_{\boldsymbol{o b j}}\right.$ y $\left.\boldsymbol{f}_{\boldsymbol{o b j 1 0}}\right)$. Estas diferencias también pueden verse reflejas en la 
Tabla 2: Medianas de Tpo. total de viaje $\left(\mathrm{TT}_{\mathrm{v}}\right)$ y $\mathbf{T p o}$. total de espera $\left(\mathrm{TT}_{\mathrm{EP}}\right)$ obtenido por las funciones propuestas para las instancias de Málaga y Paris

\begin{tabular}{|c|c|c|c|c|}
\hline \multirow[b]{2}{*}{ Función } & \multicolumn{2}{|c|}{ Málaga } & \multicolumn{2}{|c|}{ Paris } \\
\hline & $\mathbf{T T}_{\mathrm{V}}$ (seg) & TTEP $_{\text {EP }}$ & TTV (seg) & TTEP (seg) $_{\text {(s) }}$ \\
\hline$f_{\text {obj1 }}$ & 475182 & 150105 & 698440 & 301685 \\
\hline$f_{o b j 6}$ & 507789 & 187344 & 754876 & 358266 \\
\hline$f_{\text {obj } 9}$ & 478976 & 155795 & 709003 & 309511 \\
\hline$f_{\text {obj10 }}$ & 483599 & 160336 & 713212 & 317874 \\
\hline$f_{\text {obj11 }}$ & 475995 & 156205 & 709964 & 311600 \\
\hline$f_{\text {obj12 }}$ & 476820 & 155233 & 710319 & 313561 \\
\hline$f_{\text {obj13 }}$ & 479162 & 157114 & 703318 & 305562 \\
\hline $\begin{array}{c}\text { ANOVA / } \\
\text { K-W }\end{array}$ & $(\bullet)$ & $(\bullet)$ & $(\bullet)$ & $(\bullet)$ \\
\hline Holm & $f_{o b j 1} \rightarrow f_{o b j 6}$ & $\begin{array}{l}f_{o b j 1} \rightarrow f_{o b j 6}, f_{o b j 10}, \\
f_{o b j 11}, f_{o b j 13}\end{array}$ & $\begin{array}{l}f_{o b j 1} \rightarrow f_{o b j 6}, \\
f_{o b j 10}\end{array}$ & $\begin{array}{l}f_{o b j 1} \rightarrow f_{o b j 6}, \\
f_{o b j 10}\end{array}$ \\
\hline
\end{tabular}

(a) Málaga

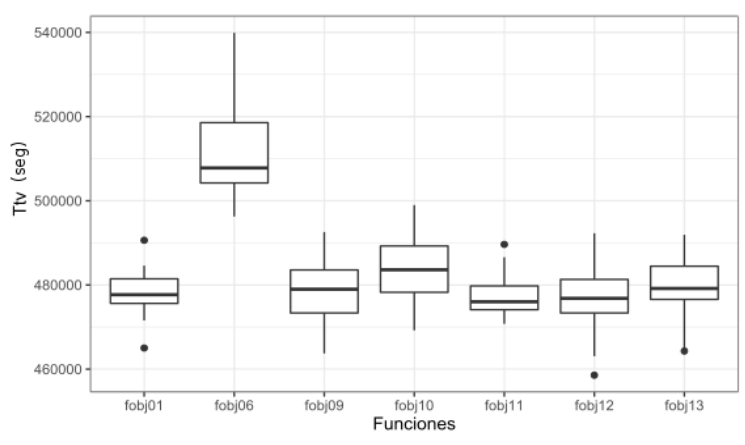

(c) Málaga

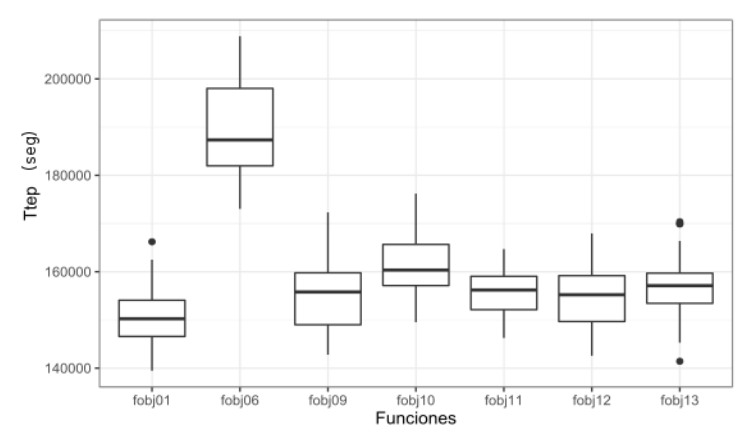

(b) Paris

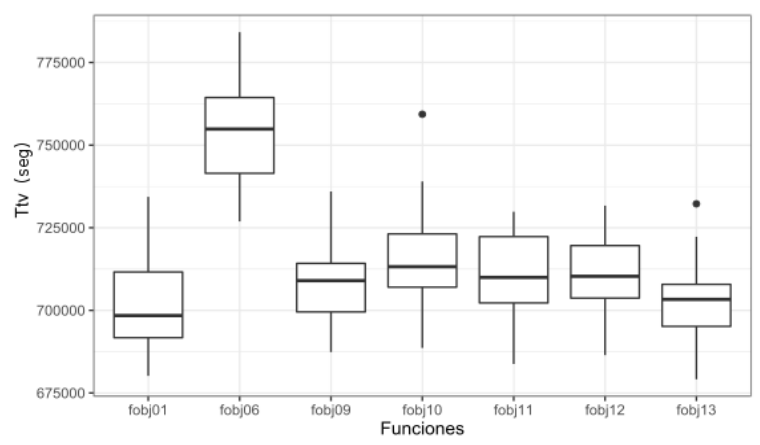

(d) Paris

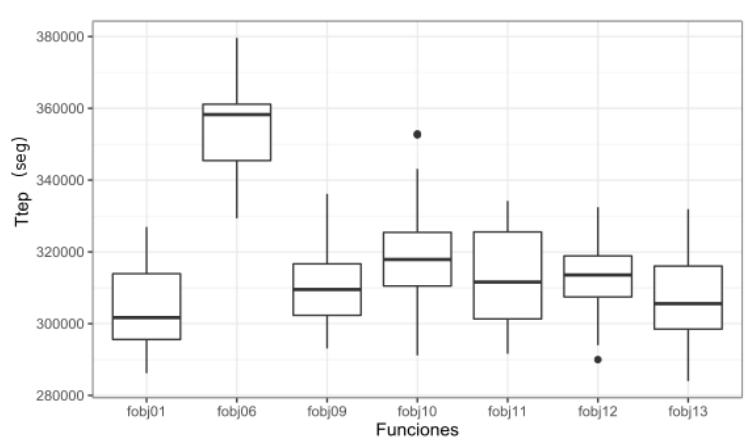

Figura 2: Boxplot de $\mathbf{T T}_{\mathrm{v}}$ y $\mathrm{TT}_{\mathrm{EP}}$ obtenido por las funciones propuestas con las instancias de Málaga y Paris

A continuación, observaremos desde otro punto de vista (tercera etapa) los resultados de las funciones, atendiendo a los gases y combustible consumido de las funciones de evaluación de calidad seleccionadas. 
Tabla 3: Mediana de gases emitidos y combustible consumido obtenido por las funciones propuestas para la instancia de Málaga

\begin{tabular}{|c|c|c|c|c|c|c|}
\hline \multirow[b]{2}{*}{ Función } & \multicolumn{6}{|c|}{ Málaga } \\
\hline & $\begin{array}{l}\mathrm{CO}_{2} \\
(\mathrm{~kg})\end{array}$ & $\begin{array}{l}\mathrm{CO} \\
(\mathrm{kg})\end{array}$ & $\begin{array}{r}\mathrm{HC} \\
(\mathrm{kg})\end{array}$ & $\begin{array}{c}\mathrm{NO}_{\mathrm{x}} \\
(\mathrm{kg})\end{array}$ & $\begin{array}{c}\mathbf{P M}_{\mathbf{x}} \\
(\mathbf{k g})\end{array}$ & $\begin{array}{c}\text { Combustible } \\
\text { (I) }\end{array}$ \\
\hline$f_{\text {obj1 }}$ & 705,41 & 5,91 & 0,93 & 4,59 & 0,10 & 280,92 \\
\hline$f_{\text {obj6 }}$ & 699,09 & 5,72 & 0,90 & 4,52 & 0,10 & 278,34 \\
\hline$f_{\text {obj } 9}$ & 696,28 & 5,77 & 0,91 & 4,52 & 0,10 & 277,27 \\
\hline$f_{\text {obj10 }}$ & 683,63 & 5,94 & 0,93 & 4,38 & 0,09 & 272,27 \\
\hline$f_{o b j 11}$ & 688,63 & 5,85 & 0,92 & 4,44 & 0,09 & 274,24 \\
\hline$f_{o b j 12}$ & 689,16 & 5,81 & 0,91 & 4,44 & 0,10 & 274,44 \\
\hline$f_{o b j 13}$ & 695,66 & 5,80 & 0,91 & 4,51 & 0,10 & 277,01 \\
\hline $\begin{array}{c}\text { Anoval } \\
K-W\end{array}$ & $(\bullet)$ & $(\bullet)$ & $(\bullet)$ & $(\bullet)$ & $(\bullet)$ & $(\bullet)$ \\
\hline Holm & $\begin{array}{l}f_{\text {obj10 }} \rightarrow \\
f_{\text {obj1 } 1, f_{\text {obj6 }}} \\
f_{\text {obj } 9, f_{o b j 13}}\end{array}$ & $\begin{array}{l}f_{o b j 6} \rightarrow \\
f_{o b j 1}, f_{o b j 10} \\
f_{o b j 11}, f_{o b j 12}\end{array}$ & $\begin{array}{l}f_{o b j 6} \rightarrow \\
f_{\text {obj1 }}, f_{o b j} 9, \\
f_{o b j 10,}, f_{o b j 11}, \\
f_{o b j 12}\end{array}$ & $\begin{array}{l}f_{o b j 10} \rightarrow \\
f_{o b j 1}, f_{o b j 6}, \\
f_{o b j 9}, f_{o b j 12,}, \\
f_{o b j 13}\end{array}$ & $\begin{array}{l}f_{\text {obj10 }} \rightarrow \\
f_{\text {obj } 1, f_{\text {obj } 6},} \\
f_{\text {obj } 9,}, f_{o b j 12}, \\
f_{\text {obj13 }}\end{array}$ & $\begin{array}{l}f_{o b j 10} \rightarrow f_{o b j 1} \\
f_{o b j 6,} f_{o b j}, \\
f_{o b j 13}\end{array}$ \\
\hline
\end{tabular}

En la Tabla 3 se pueden observar las medianas obtenidas para las emisiones de gases y el combustible consumido utilizando cada una de las funciones propuestas en la instancia de Málaga. Podemos observar que con la función $\boldsymbol{f}_{\text {obj10 }}$ se obtienen los menores valores para los gases $\mathrm{CO}_{2}, \mathrm{NO}_{\mathrm{x}}$ у $\mathrm{PM}_{\mathrm{x}},(683,63 \mathrm{~kg} ; 4,38 \mathrm{~kg} ; 0,09 \mathrm{~kg}$ respectivamente) así como también una reducción en el consumo de combustible (272, 27 litros). En cuanto a $\mathrm{CO}$ y HC los menores valores fueron obtenidos por la función $\boldsymbol{f}_{\boldsymbol{o b j}}(5,72 \mathrm{~kg}$ para $\mathrm{CO}$ y $0,90 \mathrm{~kg}$ para $\mathrm{HC})$. En todos los análisis las diferencias entre los resultados de las funciones han sido estadísticamente significativos. Podemos observar que la función $\boldsymbol{f}_{\text {obj10 }}$ tiene diferencias estadísticamente significativas con los resultados de cinco funciones $\left(f_{o b j 1}, f_{o b j 6}, f_{o b j}, f_{o b j 12}\right.$ y $\left.f_{o b j 13}\right)$ para los resultados obtenidos en cuanto a $\mathrm{NO}_{\mathrm{x}}$ y $\mathrm{PM}_{\mathrm{x}}$ y tiene diferencias estadísticamente significativas con cuatro funciones $\left(f_{\boldsymbol{o b j}}, \boldsymbol{f}_{\boldsymbol{o b j} \boldsymbol{b}}, \boldsymbol{f}_{\boldsymbol{o b j} \boldsymbol{9}}\right.$ y $\left.\boldsymbol{f}_{\boldsymbol{o b j} 13}\right)$ para $\mathrm{CO}_{2}$ y combustible. En cuanto a la función $f_{\text {obj }}$ tiene diferencias estadísticamente significativas con los resultados obtenidos de cuatro funciones $\left(f_{o b j 1}, f_{o b j 10}, f_{o b j 11}\right.$ y $\left.f_{o b j 12}\right)$ para $\mathrm{CO}$ y con respecto a $\mathrm{HC}$ tiene diferencias estadísticamente significativas con los resultados obtenidos por cinco funciones $\left(\boldsymbol{f}_{\boldsymbol{o b j}} \boldsymbol{1}, \boldsymbol{f}_{\boldsymbol{o b j} j}\right.$, $f_{\text {obj10, }} \boldsymbol{f}_{\text {obj11 }}$ y $\boldsymbol{f}_{\boldsymbol{o b j 1 2}}$ ). Observemos también que los resultados de las funciones $\boldsymbol{f}_{\boldsymbol{o b j} 10}$ y $\boldsymbol{f}_{\text {obj11 }}$ no presentan diferencias estadísticamente significativas entre ellas siendo que la función $\boldsymbol{f}_{\boldsymbol{o b j}}$ presenta en mediana menores valores.

En la Tabla 4 mostramos la misma información (medianas de gases emitidos y combustible consumido) obtenido por las funciones con la instancia de Paris. Podemos observar que con esta instancia las funciones que logran una reducción mayor en gases y combustible son tres $\left(f_{o b j 10}, f_{o b j 11}\right.$ y $\left.\boldsymbol{f}_{\boldsymbol{o b j} 13}\right)$. La función $\boldsymbol{f}_{\boldsymbol{o b j 1 0}}$ obtiene los menores valores para $\mathrm{NO}_{\mathrm{x}}$ con diferencias estadísticamente significativas con respecto a los resultados obtenidos para estas variables por

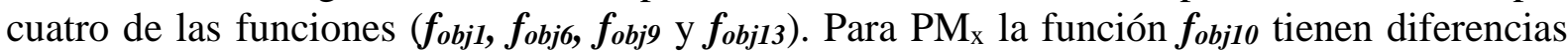
estadísticamente significativas con respecto a los resultados de cinco funciones $\left(\boldsymbol{f}_{\boldsymbol{o b j} \mathbf{1}}, \boldsymbol{f}_{\boldsymbol{o b j} \boldsymbol{}}, \boldsymbol{f}_{\boldsymbol{o b j} j}\right.$, $\boldsymbol{f}_{\boldsymbol{o b j 1 2}}$ y $\boldsymbol{f}_{\boldsymbol{o b j}}$ 13). La función $\boldsymbol{f}_{\boldsymbol{o b j} 11}$ obtiene los menores valores para $\mathrm{CO}_{2}$ y Combustible. Esta función tiene diferencias estadísticamente significativas con los resultados obtenidos por cuatro funciones $\left(\boldsymbol{f}_{\boldsymbol{o b j} \mathbf{j}}, \boldsymbol{f}_{\boldsymbol{o b j} \boldsymbol{b}}, \boldsymbol{f}_{\boldsymbol{o b j} \boldsymbol{9}}\right.$ y $\left.\boldsymbol{f}_{\boldsymbol{o b j}} \mathbf{1 3}\right)$. No existen diferencias estadísticamente significativas entre los resultados obtenidos por $\boldsymbol{f}_{\boldsymbol{o b j} 10}$ y $\boldsymbol{f}_{\boldsymbol{o b j} \mathbf{1 1}}$ en ninguna de las variables analizadas. Finalmente, la 
función $\boldsymbol{f}_{\text {obj13 }}$ obtiene los menores valores para CO y $\mathrm{HC}(7,96 \mathrm{~kg}$ y $1,22 \mathrm{~kg}$, respectivamente), presentando diferencias estadísticamente significativas con respecto a cuatro funciones $\left(f_{o b j l}\right.$, $f_{o b j 10,} f_{o b j 11}$ y $\left.f_{o b j 12}\right)$.

Tabla 4: Mediana de gases emitidos y combustible obtenido por las funciones propuestas para la instancia de Paris

\begin{tabular}{|c|c|c|c|c|c|c|}
\hline \multirow[b]{2}{*}{ Función } & \multicolumn{6}{|c|}{ Paris } \\
\hline & $\begin{array}{l}\mathrm{CO}_{2} \\
(\mathrm{~kg})\end{array}$ & $\begin{array}{l}\mathrm{CO} \\
(\mathrm{kg})\end{array}$ & $\begin{array}{l}\mathrm{HC} \\
(\mathrm{kg})\end{array}$ & $\begin{array}{c}\mathrm{NO}_{\mathrm{x}} \\
(\mathrm{kg})\end{array}$ & $\begin{array}{c}\mathbf{P M}_{\mathbf{x}} \\
(\mathbf{k g})\end{array}$ & $\begin{array}{c}\text { Combustible } \\
\text { (l) }\end{array}$ \\
\hline$f_{\text {obj1 }}$ & 903,72 & 8,16 & 1,25 & 5,92 & 0,13 & 359,57 \\
\hline$f_{\text {obj6 }}$ & 909,96 & 8,03 & 1,23 & 5,97 & 0,13 & 362,01 \\
\hline$f_{o b j g}$ & 898,74 & 7,99 & 1,23 & 5,88 & 0,13 & 357,56 \\
\hline$f_{\text {obj10 }}$ & 890,80 & 8,32 & 1,26 & 5,75 & $\mathbf{0 , 1 3}$ & 354,48 \\
\hline$f_{o b j 11}$ & 889,77 & 8,18 & 1,24 & 5,77 & 0,13 & 354,03 \\
\hline$f_{o b j 12}$ & 893,70 & 8,08 & 1,24 & 5,82 & 0,13 & 355,64 \\
\hline$f_{\text {obj } 13}$ & 895,38 & 7,96 & 1,22 & 5,87 & 0,13 & 356,28 \\
\hline $\begin{array}{c}\text { Anoval } \\
K-W\end{array}$ & $(\bullet)$ & $(\bullet)$ & $(\bullet)$ & $(\bullet)$ & $(\bullet)$ & $(\bullet)$ \\
\hline Holm & $\begin{array}{l}f_{\text {obj11 }} \longrightarrow \\
f_{\text {obj1 }}, f_{\text {obj } 6}, \\
f_{\text {obj } 9, f_{\text {obj } 13}}\end{array}$ & $\begin{array}{l}f_{o b j 13} \rightarrow \\
f_{o b j 1}, f_{o b j 10}, \\
f_{o b j 11}, f_{o b j 12}\end{array}$ & $\begin{array}{l}f_{o b j 13} \rightarrow \\
f_{o b j 1}, f_{o b j 10}, \\
f_{o b j 11}, f_{o b j 12}\end{array}$ & $\begin{array}{l}f_{o b j 10} \rightarrow \\
f_{o b j 1}, f_{\text {obj6 }}, \\
f_{o b j 9,} f_{o b j 13}\end{array}$ & 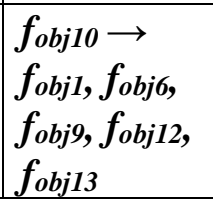 & $\begin{array}{l}f_{\text {obj11 }} \longrightarrow f_{\text {obj1 } 1}, \\
f_{\text {obj6, }} f_{\text {obj }}, \\
f_{\text {obj13 }}\end{array}$ \\
\hline
\end{tabular}

Por tema de espacio únicamente colocaremos algunas gráficas de boxplot en representación del análisis de estas variables (gases y combustible).

(a)Málaga

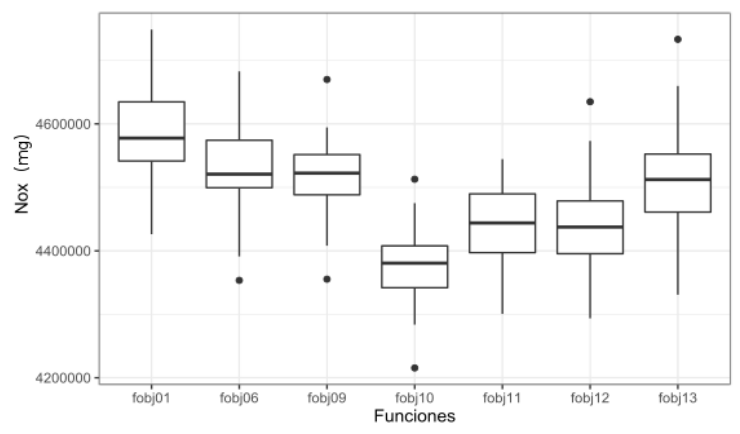

(c)Málaga

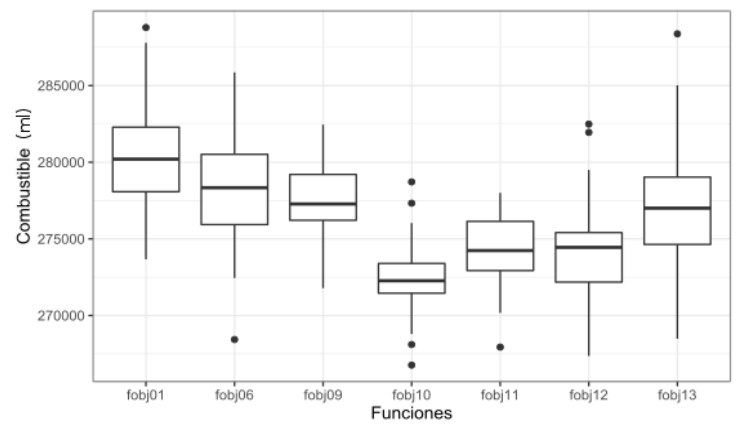

(b)Paris

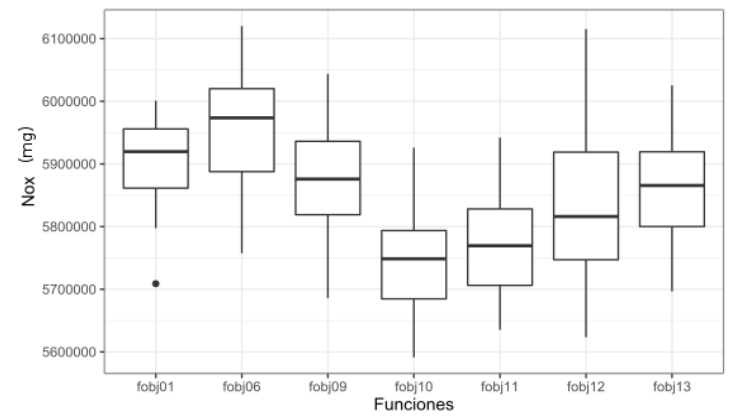

(d)Paris

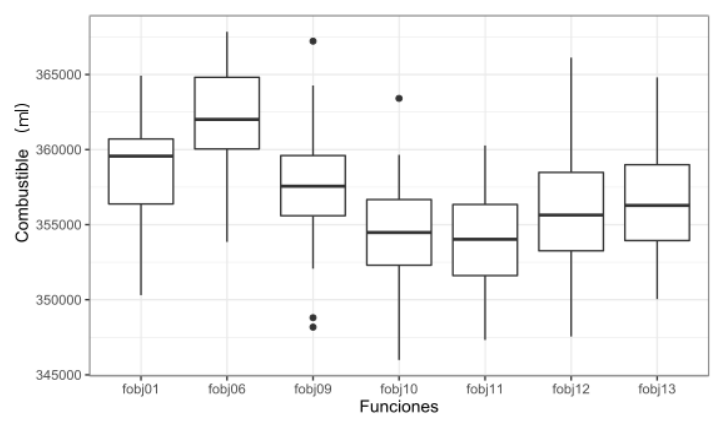

Figura 3: Medianas de emisiones de $\mathrm{NO}_{\mathrm{x}} \mathrm{y}$ consumo de combustible obtenido por las funciones propuestas para las instancias de Málaga y de Paris 
La Figura 3 muestra los resultados de emisiones de $\mathrm{NO}_{\mathrm{x}}$ y consumo de combustible obtenidas por las funciones propuestas para las instancias de Málaga y de Paris. Podemos observar cómo se distribuyan los resultados de cada una de las funciones alrededor de la mediana y también las diferencias estadísticamente significativas se ven reflejas en las gráficas. Se puede visualizar una clara diferencia en los resultados obtenidos por la función $\boldsymbol{f}_{\text {obj10 }}$ para la instancia de Málaga en cuanto a $\mathrm{PM}_{\mathrm{x}}$ y consumo de combustible (Figura 3 (a) y (c)). En cuanto a los resultados obtenidos para la instancia de Paris vemos que tanto $\boldsymbol{f}_{\text {obj10 }}$ y $\boldsymbol{f}_{\text {objl1 }}$ obtienen los menores valores (Figura 3 (b) y (d)) y no tienen diferencias estadísticamente significativas entre ellas.

El siguiente análisis corresponde a la etapa cuatro donde se toman las funciones que han tenido los mejores resultados tanto analizando las variables de tránsito como los gases y combustible. Por lo tanto, compararemos los resultados obtenidos de las funciones $\boldsymbol{f}_{\text {obj }}, \boldsymbol{f}_{\boldsymbol{o b j} 10}$ y $\boldsymbol{f}_{\boldsymbol{o b j 1 1}}$ (Tabla 5 y Tabla 6). Podemos observar en la Tabla 5 que para las variables de tránsito ( $\mathrm{TT}_{\mathrm{v}}$ y $\mathrm{TT}_{\mathrm{EP}}$ ) los menores valores son obtenidos por la función $\boldsymbol{f}_{\boldsymbol{o b j} 1}$ y que las diferencias son estadísticamente significativas entre los resultados de $\boldsymbol{f}_{\boldsymbol{o b j} 1}$ y $\boldsymbol{f}_{\boldsymbol{o b j} \mathbf{1 0}}$. En cuanto a los gases y combustible podemos observar que la función $\boldsymbol{f}_{\text {obj10 }}$ es la que presenta la menor emisión de tres gases analizados $\left(\mathrm{CO}_{2}\right.$, $\mathrm{NO}_{\mathrm{x}}$ y $\mathrm{PMx}$ ) con diferencias estadísticamente significativas con respecto a las otras dos funciones $\left(\boldsymbol{f}_{\boldsymbol{o b j}} \mathbf{y} \boldsymbol{f}_{\boldsymbol{o b j}} \boldsymbol{1}\right)$ para la instancia de Málaga.

Tabla 5: Resultados obtenidos con la aplicación de las funciones $f_{o b j l}, f_{o b j 10}$ y $f_{o b j 11}$ en la instancia de Málaga para todas las variables de análisis

\begin{tabular}{|c|c|c|c|c|c|c|c|c|}
\hline \multirow[b]{2}{*}{ Función } & \multicolumn{8}{|c|}{ Málaga } \\
\hline & $\begin{array}{l}\mathbf{T T}_{\mathbf{v}} \\
\text { (seg) }\end{array}$ & $\begin{array}{c}\text { TTEP } \\
\text { (seg) }\end{array}$ & $\begin{array}{c}\mathrm{CO}_{2} \\
(\mathrm{~kg})\end{array}$ & $\begin{array}{l}\text { CO } \\
\text { (kg) }\end{array}$ & $\begin{array}{l}\mathrm{HC} \\
(\mathrm{kg})\end{array}$ & $\begin{array}{c}\mathrm{NO}_{\mathrm{x}} \\
(\mathrm{kg})\end{array}$ & $\begin{array}{c}\mathbf{P M}_{\mathbf{x}} \\
(\mathrm{kg})\end{array}$ & $\begin{array}{c}\text { Comb. } \\
\text { (l) }\end{array}$ \\
\hline$f_{o b j 1}$ & 475182 & 150105 & 705,41 & 5,91 & 0,93 & 4,59 & 0,10 & 280,92 \\
\hline$f_{\text {objlo }}$ & 483599 & 160336 & 683,63 & 5,94 & 0,93 & 4,38 & 0,09 & 272,27 \\
\hline$f_{\text {obj11 }}$ & 475995 & 156205 & 688,63 & 5,85 & 0,92 & 4,44 & 0,09 & 274,24 \\
\hline $\begin{array}{c}\text { Anoval } \\
K-W\end{array}$ & $(\bullet)$ & $(\bullet)$ & $(\bullet)$ & $(\bullet)$ & $(\bullet)$ & $(\bullet)$ & $(\bullet)$ & $(\bullet)$ \\
\hline Holm & $\begin{array}{l}f_{\text {obj1 } 1} \rightarrow \\
f_{\text {obj10 }}\end{array}$ & $\begin{array}{l}f_{o b j 1} \longrightarrow \\
f_{o b j 10}, \\
f_{o b j 11}\end{array}$ & $\begin{array}{l}f_{\text {obj10 }} \rightarrow \\
f_{\text {obj1 }}, \\
f_{\text {obj11 }}\end{array}$ & $\begin{array}{l}f_{\text {obj11 }} \rightarrow \\
f_{\text {objl }}, \\
f_{\text {obj10 }} \\
\end{array}$ & $\begin{array}{l}f_{\text {obj11 }} \longrightarrow \\
f_{\text {obj1 }}, \\
f_{\text {obj10 }}\end{array}$ & $\begin{array}{l}f_{\text {obj10 }} \rightarrow \\
f_{\text {obj1 }}, \\
f_{\text {obj11 }}\end{array}$ & $\begin{array}{l}f_{\text {obj10 }} \rightarrow \\
f_{\text {obj1 }}, \\
f_{\text {obj } 11}\end{array}$ & $\begin{array}{l}f_{\text {obj10 }} \rightarrow \\
f_{\text {obj1 }}, \\
f_{\text {obj11 }}\end{array}$ \\
\hline
\end{tabular}

Tabla 6: Resultados obtenidos con la aplicación de las funciones $f_{o b j l}, f_{o b j l o}$ y $f_{o b j 11}$ en la instancia de Paris para todas las variables de análisis

\begin{tabular}{|c|c|c|c|c|c|c|c|c|}
\hline \multirow[b]{2}{*}{ Función } & \multicolumn{8}{|c|}{ Paris } \\
\hline & $\begin{array}{l}\mathbf{T T}_{\mathbf{v}} \\
\text { (seg) }\end{array}$ & $\begin{array}{l}\text { TT }_{\text {EP }} \\
\text { (seg) }\end{array}$ & $\begin{array}{c}\mathrm{CO}_{2} \\
(\mathrm{~kg})\end{array}$ & $\begin{array}{l}\text { CO } \\
(\mathrm{kg})\end{array}$ & $\begin{array}{l}\mathrm{HC} \\
(\mathrm{kg})\end{array}$ & $\begin{array}{c}\mathrm{NO}_{\mathbf{x}} \\
(\mathrm{kg})\end{array}$ & $\begin{array}{c}\mathbf{P M}_{\mathbf{x}} \\
(\mathrm{kg})\end{array}$ & $\begin{array}{c}\text { Comb. } \\
\text { (l) }\end{array}$ \\
\hline$f_{\text {obj1 }}$ & 698440 & 301685 & 903,72 & 8,16 & 1,25 & 5,92 & 0,13 & 359,57 \\
\hline$f_{o b j 10}$ & 713212 & 317874 & 890,80 & 8,32 & 1,26 & 5,75 & 0,13 & 354,48 \\
\hline$f_{o b j 11}$ & 709964 & 311600 & 889,77 & 8,18 & 1,24 & 5,77 & 0,13 & 354,03 \\
\hline $\begin{array}{c}\text { Anoval } \\
K-W\end{array}$ & $(\bullet)$ & $(\bullet)$ & $(\bullet)$ & $(\bullet)$ & $(\bullet)$ & $(\bullet)$ & $(\bullet)$ & $(\bullet)$ \\
\hline Holm & $\begin{array}{l}f_{o b j 1} \longrightarrow \\
f_{o b j 10}\end{array}$ & $\begin{array}{l}f_{\text {obj1 }} \rightarrow \\
f_{\text {obj10 }}\end{array}$ & $\begin{array}{l}f_{o b j 10,} \\
f_{o b j 11} \\
\rightarrow f_{o b j 1}\end{array}$ & $\begin{array}{l}f_{o b j 1}, \\
f_{o b j 11} \\
\rightarrow f_{o b j 10}\end{array}$ & $\begin{array}{l}f_{\text {obj11 }} \rightarrow \\
f_{\text {obj1 }}, \\
f_{\text {obj10 }}\end{array}$ & $\begin{array}{l}f_{o b j 10,} \\
f_{o b j 11} \\
\rightarrow f_{o b j 1}\end{array}$ & $\begin{array}{l}f_{o b j 10,} \\
f_{o b j 11} \\
\rightarrow f_{o b j 1}\end{array}$ & $\begin{array}{l}f_{o b j 10} \\
f_{o b j 11} \\
\rightarrow f_{o b j 1}\end{array}$ \\
\hline
\end{tabular}


En la Tabla 6 podemos observar que para las variables de tránsito $\left(\mathrm{TT}_{\mathrm{v}} \mathrm{y} \mathrm{TT}_{\mathrm{EP}}\right)$ los menores valores son obtenidos por la función $\boldsymbol{f}_{\text {objl }}$ y de igual forma que con la instancia de Málaga las diferencias son estadísticamente significativas entre los resultados de $\boldsymbol{f}_{\boldsymbol{o b j} \mathbf{1}}$ y $\boldsymbol{f}_{\boldsymbol{o b j} \mathbf{1 0}}$. Con respecto a las otras variables de análisis las funciones $\boldsymbol{f}_{\boldsymbol{o b j 1 0}} y \boldsymbol{f}_{\boldsymbol{o b j 1 1}}$ producen una reducción en el consumo de combustible y en la emisión de la mayoría de los gases $\left(\mathrm{CO}_{2}, \mathrm{HC}, \mathrm{NO}_{\mathrm{x}}, \mathrm{PM}_{\mathrm{x}}\right)$, ambas con diferencias estadísticamente significativas con respecto a los resultados obtenidos por la función $\boldsymbol{f}_{\boldsymbol{o b j} \mathbf{1}}$. Está última función redujo la emisión de $\mathrm{CO}$ solamente con diferencias estadísticamente significativas con respecto a $\boldsymbol{f}_{\text {objlo }}$.

En la Figura 4 mostramos las gráficas de boxplot para los resultados obtenidos para $\mathrm{CO}_{2}$ y $\mathrm{PM}_{\mathrm{x}}$ para ambas instancias (Málaga y Paris). Podemos observar claramente como se alejan las medianas obtenidas para $\mathrm{CO}_{2}$ de la función $\boldsymbol{f}_{\boldsymbol{o b j l}}$ con respecto a las otras dos funciones (Figura 4 (a) y (b)) y los valores casi idénticos de las medianas de $\mathrm{CO}_{2}$ para $\boldsymbol{f}_{\boldsymbol{o b j} 10}$ y $\boldsymbol{f}_{\text {obj11 }}$ en la instancia de Paris (Figura 4 (b)). Se puede observar claramente entre que funciones existen las diferencias estadísticamente significativas. También se pueden visualizar estas diferencias para los resultados obtenidos con respecto a $\mathrm{PM}_{\mathrm{x}}$ (Figura 4 (c) y (d)) para ambas instancias.

(a)Málaga

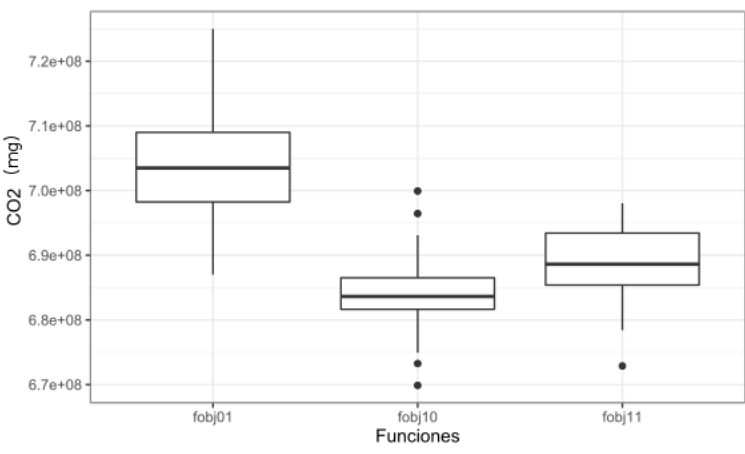

(c)Málaga

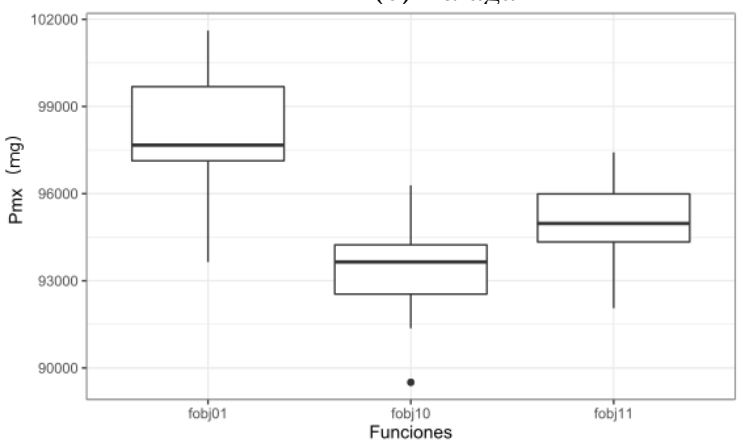

(b)Paris

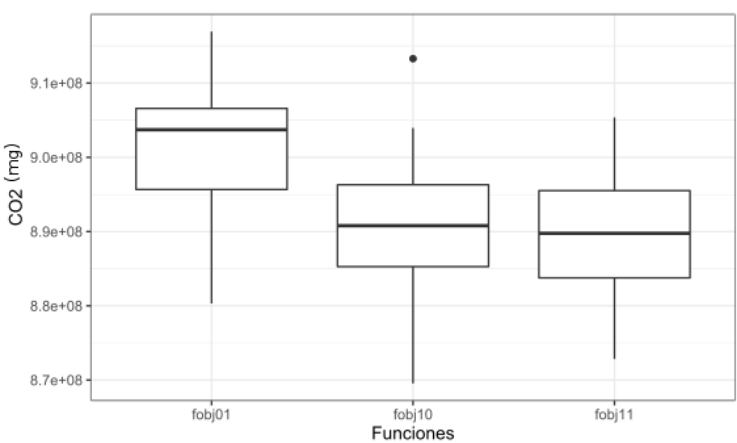

(d)Paris

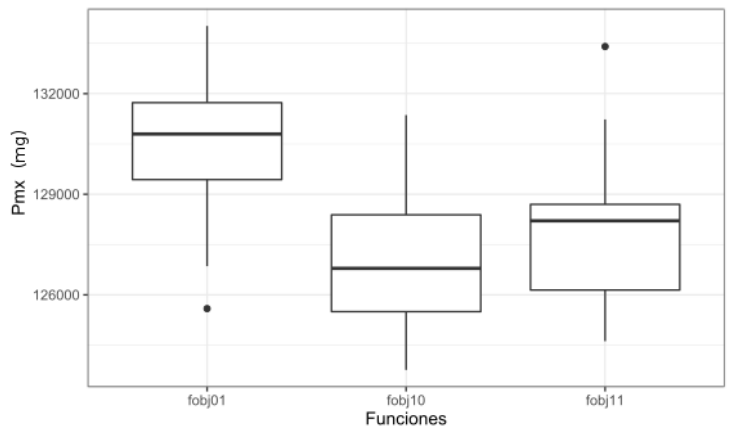

Figura 4: Medianas de gases $\left(\mathrm{CO}_{2}\right.$ y $\left.\mathrm{PM}_{\mathrm{x}}\right)$ obtenidos de la aplicación de las funciones $\boldsymbol{f}_{\boldsymbol{o b j}}$, $\boldsymbol{f}_{\boldsymbol{o b j} 10}$ y $\boldsymbol{f}_{\boldsymbol{o b j 1 1}}$ con las instancias de Málaga y Paris

\section{CONCLUSIONES}

Este trabajado se ha centrado en el estudio de diferentes propuestas de funciones de optimización utilizadas como motor de búsqueda para cGA. Se utiliza como base una función objetivo $\left(f_{o b j l}\right)$ que ha tenido muy buenos resultados en trabajos previos y está formada por diferentes variables de tránsito a optimizar. Como nuestro objetivo es lograr no solamente una mejora en el tránsito sino también una reducción en la emisión de gases y consumo de 
combustible se proponen nuevas funciones (14 funciones de evaluación de calidad que combinan variables de tránsito, gases y combustible consumido).

De las funciones propuestas se descartan del análisis aquellas funciones que no lograron que todos los vehículos alcancen su destino.

De los análisis realizados observando primero los resultados obtenidos en cuanto a variables de tránsito Tiempo total de viaje de los vehículos y Tiempo de paradas y espera de todos los vehículos la función objetivo que obtiene los mejores resultados para las instancias de Málaga y Paris es la función $\boldsymbol{f}_{\text {obj }}$. No obstante, si queremos lograr una reducción en el consumo de combustible y en la emisión de gases hemos comprobado con diferencias estadísticamente significativas que las funciones $\boldsymbol{f}_{\boldsymbol{o b j} 10}$ y $\boldsymbol{f}_{\boldsymbol{o b j} 11}$ obtienen reducciones entre un $1.5 \%$ y un $2.88 \%$ de

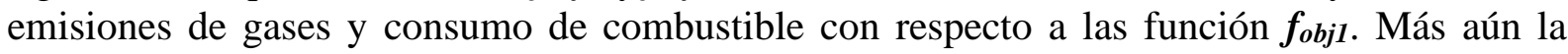
función $\boldsymbol{f}_{\text {obj11 }}$ además presenta un muy buen desempeño en cuanto a las reducción en tiempo de variables de tránsito (los resultados obtenidos no presentan diferencias estadísticamente significativas con respecto a $\boldsymbol{f}_{\boldsymbol{o b j} \mathbf{1}}$ ) tanto para a instancia de Málaga como para la de Paris. Se ha demostrado que la función de evaluación de calidad $f_{\text {obj11 }}$ es adecuada no solamente para el tráfico sino también para reducir las emisiones de gases y consumo de combustible.

Trabajos futuros se centrarán en reducir el número de evaluaciones con el objetivo de reducir el esfuerzo computacional en términos de tiempo de procesamiento. Incorporar funciones multiobjetivo e incrementar la cantidad de instancias de otras ciudades en particular de Argentina.

\section{AGRADECIMIENTOS}

Este trabajo ha sido parcialmente financiado por el proyecto 29/B225 "Soluciones inteligentes para el desarrollo urbano sostenible". Los autores agradecen a la Universidad Nacional de la Patagonia Austral.

\section{REFERENCIAS}

BASKAN, O., y HALDENBILEN, S. (2011). Ant colony optimization approach for optimizing traffic signal timings. In Ant colony optimization-methods and applications. IntechOpen.

BASKAN, O., HALDENBILEN, S., CEYLAN, H., y CEYLAN, H. (2009). A new solution algorithm for improving performance of ant colony optimization. Applied Mathematics and Computation, 211(1), 75-84.

CEYLAN H. y BELL. M. Traffic signal timing optimisation based on genetic algorithm approach, including drivers? routing. Transportation Research Part B: Methodological, 38(4):329-342, 2004.

DEZANI, H., GOMES, L., DAMIANI, F., y MARRANGHELLO, N. (2012). Controlling traffic jams on urban roads modeled in Coloured Petri net using Genetic Algorithm. In IECON 2012-38th Annual Conference on IEEE Industrial Electronics Society (pp. 3043-3048). IEEE.

DORRONSORO, B., y ALBA, E. (2008). Cellular Genetic Algorithms (Vol. 42). SpringerVerlGA US. 
GARCÍA-NIETO, J., ALBA, E., y OLIVERA, A. C. (2012). Swarm intelligence for traffic light scheduling: Application to real urban areas. Engineering Applications of Artificial Intelligence, 25(2), 274-283.

GARCIA-NIETO, J., OLIVERA, A. C., y ALBA, E. (2013). Optimal cycle program of traffic lights with particle swarm optimization.

GARCIA-NIETO, J., FERRER J., y ALBA E. (2014). Optimising traffic lights with metaheuristics: Reduction of car emissions and consumption. In Neural Networks (IJCNN), 2014 International Joint Conference on, pages 48-54. IEEE, 2014.

JABBARPOUR, M. R., MALAKOOTI, H., NOOR, R. M., ANUAR, N. B., y KHAMIS, N. (2014). Ant colony optimisation for vehicle traffic systems: applications and challenges. International Journal of Bio-Inspired Computation, 6(1), 32-56.

JUN, Z., MEI-JING, G., MEI-NA, S., JUN-TAO, X., ZHENG-LING, Y., y XIAO-DONG, L. (2012). Port traffic signal control method based on gradient descent and pso algorithm. In Proceedings of the 31st Chinese Control Conference (pp. 7338-7342). IEEE.

KRAJZEWICZ, D., BONERT, M., y WAGNER, P. (2006). The open source traffic simulation package SUMO. RoboCup 2006.

KUN, C. H. E. N., y LEI, Y. U. (2007). Microscopic traffic-emission simulation and case study for evaluation of traffic control strategies. Journal of Transportation Systems Engineering and Information Technology, 7(1), 93-99.

NESMACHNOW, S., MASSOBRIO, R., ARRECHE, E., MUMFORD, C., OLIVERA, A. C., VIDAL, P. J., y TCHERNYKH, A. (2019). Traffic lights synchronization for Bus Rapid Transit using a parallel evolutionary algorithm. International Journal of Transportation Science and Technology, 8(1), 53-67.

OLIVERA, A. C., GARCÍA-NIETO, J. M., y ALBA, E. (2015). Reducing vehicle emissions and fuel consumption in the city by using particle swarm optimization. Applied Intelligence, 42(3), 389-405.

PÉRES, M., RUIZ, G., NESMACHNOW, S., \& OLIVERA, A. C. (2018). Multiobjective evolutionary optimization of traffic flow and pollution in Montevideo, Uruguay. Applied Soft Computing, 70, 472-485.

SÁNCHEZ, J., GALÁN, M., y RUBIO, E. (2008). Applying a traffic lights evolutionary optimization technique to a real case:"Las Ramblas" area in Santa Cruz de Tenerife. IEEE Transactions on evolutionary computation, 12(1), 25-40.

SOURAVLIAS, D., LUQUE, G., ALBA, E., y PARSOPOULOS, K. E. (2016). Smart traffic lights: A first parallel computing approach. In 2016 International Conference on Intelligent Networking and Collaborative Systems (INCoS) (pp. 229-236). IEEE.

THAIN, D., TANNENBAUM, T., Y LIVNY, M. (2005). Distributed computing in practice: the Condor experience. Concurrency and computation: practice and experience, 17(24), 323-356.

VILLAGRA, A., ALBA, E., y LUQUE, G. (2020). A better understanding on traffic light scheduling: New cellular GAs and new in-depth analysis of solutions. Journal of Computational Science, 41, 101085.

ZHOU, Z., y CAI, M. (2014). Intersection signal control multi-objective optimization based on genetic algorithm. Journal of Traffic and Transportation Engineering (English Edition), 1(2), 153-158. 